Bulletin de la Société Royale des Sciences, Vol. 89, Articles, 2020, p. 186 - 197

\title{
About Lie-Rinehart superalgebras
}

\author{
Manuscrit reçu le 31 juillet 2020 et accepté le 25 octobre 2020
}

\begin{abstract}
Claude Roger ${ }^{a}$
${ }^{a}$ Institut Camille Jordan ${ }^{1}$, Université de Lyon, Université Lyon I, 43 boulevard du 11 novembre 1918, F-69622 Villeurbanne Cedex, France
\end{abstract}

Keywords:Supermanifolds, Lie-Rinehart algebras, Frölicher-Nijenhuis bracket, Nijenhuis-Richardson bracket, Schouten-Nijenhuis bracket.

Mathematics Subject Classification (2010): 17B35, 17B66, 58A50

Abstract: We extend to the superalgebraic case the theory of Lie-Rinehart algebras and work out some examples concerning the most popular samples of supermanifolds.

\section{Introduction}

The goal of this article is to discuss some properties of Lie-Rinehart structures in a superalgebraic context. Let's first sketch the classical case; a Lie-Rinehart algebra is a couple $(A, L)$ where $L$ is a Lie algebra on a base field $k, A$ an associative and commutative $k$-algebra, with two operations

1. $(A, L) \rightarrow L$ denoted by $(a, X) \rightarrow a X$, which induces a $A$-module structure on $L$, and

2. $L \rightarrow \operatorname{Der}(A)$ denoted as $(X, a) \rightarrow L_{X}(a)$.

One further axiom is then compatibility condition between those two operations, given by the following formula:

3. $[X, a Y]=L_{X}(a) Y+a[X, Y]$

To any $(A, L)$ one can associate its enveloping algebra $\mathcal{U}(A, L)[6,7,8]$.

A canonical example of Lie-Rinehart structure is associated to any differentiable manifold $V$; let $A=\mathcal{O}_{V}$ be the algebra of smooth functions on $V$ and $L=V e c t(V)$ the Lie algebra of vector fields on $V$, operation (2) being then the Lie derivative of

\footnotetext{
${ }^{1}$ Laboratoire associé au CNRS UMR 5208
} 
functions and (1) the natural multiplication of a vector field by a function. In fact, the general definition was deduced by Rinehart [17] from an algebraic generalization of that geometric situation. For $(A, L)=\left(\mathcal{O}_{V}, V e c t(V)\right)$, the enveloping algebra is isomorphic to the associative algebra of differential operators on $V$, as we shall see in the next part.

Poisson algebras provide another standard example of Lie-Rinehart structures: let $(A,\{\}$,$) be a Poisson algebra, then the pair (A, A)$ where the first $A$ is plainly the algebra with associative multiplication, and the second one is the Lie algebra given by Poisson bracket, operation (1) being the multiplication, and (2) being defined as $L_{X}(a)=\{X, a\}$; axiom (3) is then a straightforward consequence axiom of Leibniz derivation for Poisson algebras.

Those Lie-Rinehart structures have been the subject of extensive studies, linked with symplectic geometry, Poisson structures, Lie groupoids and algebroids [18], and various kinds of quantizations; cf.[6] for a very extensive survey of those topics.

\section{Construction of the enveloping algebra}

We shall give below a short sketch of the classical construction, following Hübschmann[6]. Let $(A, L)$ be a Lie-Rinehart algebra on base field $k$, let $\mathcal{U}_{k}(L)$ be the enveloping algebra of the Lie algebra $L$ and $i_{L}: L \rightarrow \mathcal{U}_{k}(L)$ the canonical map; set $\bar{X}=i_{L}(X)$ and consider $A \otimes_{k} \mathcal{U}_{k}(L)$ with multiplication $m$ defined on generators as follows:

$$
\begin{gathered}
m(a, \bar{X})=a \otimes \bar{X} \\
m(\bar{X}, a)=a \otimes \bar{X}+L_{X}(a)
\end{gathered}
$$

Axioms of Lie-Rinehart algebras guarantee associativity; one can give a more explicit formula for the product. For $u \in \mathcal{U}_{k}(L)$, let $\Delta(u)=\sum u_{i}^{\prime} \otimes u_{i}^{\prime \prime}$ be its coproduct in $\mathcal{U}_{k}(L)$, then our algebra structure on $A \otimes_{k} \mathcal{U}_{k}(L)$ is given by the following formula:

$$
m(a \otimes u, b \otimes v)=a b \otimes u v+\sum a L_{u_{i}^{\prime}}(b) \otimes u_{i}^{\prime \prime} v .
$$

The key fact is that multiplication in $A$ is compatible with its $\mathcal{U}_{k}(L)$-module structure; analogous structures can be found in the Hopf algebras of algebraic topology (MasseyPeterson algebras), cf. [6].

One then considers the two-sided ideal $J \subset A \otimes_{k} \mathcal{U}_{k}(L)$ generated by elements $a b \otimes \bar{X}-a \otimes b \bar{X}$. One checks $(a b \otimes \bar{X}-a \otimes b \bar{X})(c \otimes \bar{Y})=a b c \otimes \bar{X} \bar{Y}+a b L_{X}(c) \otimes Y-$ $a c \otimes b \bar{X} \bar{Y}-a L_{b X}(c) \otimes Y=a b c \otimes \bar{X} \bar{Y}-a c \otimes b \bar{X} \bar{Y}$, which lives in $J$; an analogous and 
straightforward computation works for the product on the left. Finally, the enveloping algebra of $(A, L)$ is defined as the quotient:

$$
\mathcal{U}(A, L)=A \otimes_{k} \mathcal{U}_{k}(L) / J .
$$

As an example it is easy to check, as announced above, that for any manifold $V$

$$
\mathcal{U}\left(\mathcal{O}_{V}, \operatorname{Vect}(V)\right)=\operatorname{Diff}(V)
$$

the associative algebra of differential operators on $V$.

Remark: So Rinehart's theory gives a direct and elegant way of dealing with the irritating problem of relations between differential operators on a manifold and the enveloping algebra of Lie algebra of vector fields, $V e c t(V)$ being viewed as differential operators of order 1 while semi-direct product $V \operatorname{ect}(V) \ltimes \mathcal{O}_{V}$ gives differential operators of order $\leq 1$. One checks that $\operatorname{Dif} f(V)$ is a non trivial quotient of $\mathcal{U}\left(V e c t(V) \ltimes \mathcal{O}_{V}\right)$.

Moreover, $\mathcal{U}(A, L)$ inherits from $\mathcal{U}_{k}(L)$ a filtration by tensor degree, and PoincaréBirkhoff-Witt theorem enables to identify the graded algebra associated with that filtration: from $\operatorname{gr} \mathcal{U}_{k}(L)=S_{k}^{*}(L)$ one deduces $\operatorname{gr} \mathcal{U}(A, L)=S_{A}^{*}(L)$, the symmetric algebra on $L$ viewed as a $A$-module. In the case where $(A, L)=\left(\mathcal{O}_{V}, V e c t(V)\right)$, one then recovers the well-known fact that $g r \operatorname{Dif} f(V)=S_{\mathcal{O}_{V}}^{*}(V e c t(V))$, the algebra of symbols of differential operators, or more geometrically, the space of symmetric tensor fields on $V$. We have found here the link with Poisson geometry, since those tensor fields are nothing but polynomial functions on the symplectic manifold $T^{*} V$, equipped with the Liouville form.

\section{$3 \quad$ Lie-Rinehart superalgebras}

The description of the historical foundation of superalgebra and supergeometry can be found in[2], for a detailed introduction with basic definitions, cf.[4]. The definition is very natural: a Lie-Rinehart superalgebra is a couple $(A, L)$ where $L$ is a Lie superalgebra and $A$ is an associative and super commutative algebra; recall that supercommutativity reads as $a b=(-1)^{|a||b|} b a$ following Quillen (or Koszul) rule. Axioms (1) and (2) can be generalized word for word to the super case, the mappings must respect the graduations, i.e. $|a X|=|a|+|X|$ and $\left|L_{X}(a)\right|=|a|+|X|$. The key modification appears for axiom (3) in the following form :

$$
\left(3^{\prime}\right)[X, a Y]=L_{X}(a) Y+(-1)^{|a||X|} a[X, Y]
$$

(Warning: each element in $L$ and $A$ can have its own independent graduation). See[3] for some results about such structures. 
The construction of the enveloping Lie algebra $U(A, L)$ can be straightforwardly generalized to the super case; in particular one has the graded version of PoincaréBirkhoff-Witt theorem with $\operatorname{gr} \mathcal{U}(A, L)=S_{A}^{* \text {,super }}(L)$. But one has to be careful, since supersymmetric tensor product $S^{*, \text { super }}$ mean symmetric on even terms, and antisymmetric on odd ones; in other words, if $E$ splits according to parity, as $E=E_{\text {even }} \oplus E_{\text {odd }}$, then $S^{* \text { super }} E=S^{*} E_{\text {even }} \otimes \Lambda^{*} E_{\text {odd }}$.

We shall describe now the most popular examples of supermanifolds, and we shall further investigate them from the point of view of Lie-Rinehart structures.

Let $W$ be any supermanifold, let $\mathcal{O}_{W}$ be its super algebra of functions (the "structural sheaf" of $W$ ); the Lie superalgebra of tangent vector fields on $W$, by analogy with the classical case, will then be defined as the Lie superalgebra of graded derivations of $\mathcal{O}_{W}$, so $\operatorname{Vect}(W)=\operatorname{Der}\left(\mathcal{O}_{W}\right)$. We shall investigate pairs $\left(\mathcal{O}_{W}, L\right)$, where $L \subset V e c t(W)$ is both a sub Lie superalgebra, and $\mathcal{O}_{W}$-submodule of $V e c t(W)$.

From any differentiable manifold $V$ various supermanifold structures can be obtained using different sheaves of associative and graded commutative algebras, which will define various superizations of $V$, by the sheaves of their superfunctions :

1. The supermanifold $\tilde{V}$ has as structural sheaf $\mathcal{O}_{\tilde{V}}=\Omega_{*}(V)$, the sheaf of contravariant tensor fields on $V$;

2. The supermanifold $\hat{V}$ has as structural sheaf $\mathcal{O}_{\hat{V}}=\Omega^{*}(V)$, the sheaf of differential forms on $V$;

3. More generally, one associates to any vector bundle $\mathcal{E} \rightarrow V$ a supermanifold $V_{\mathcal{E}}$, with structural sheaf the sheaf of sections $\Gamma\left(V, \Lambda^{*} \mathcal{E}^{*}\right)$.

A well known theorem of M.Batchelor[1] implies that in the differentiable case all supermanifold structures can be deduced from case 3. Our examples above are obtained from $\mathcal{E}=\tau V$ and $\mathcal{E}=\tau^{\prime} V$, the tangent and cotangent bundle to $V$ respectively.

\section{The purely odd supermanifold of dimension $0 \mid n$}

We shall begin with the purely odd case, which is easier, since it reduces to pure algebra; so, let $E$ be an $n$-dimensional vector space considered as a purely odd supermanifold of dimension $0 \mid n$, the underlying differentiable manifold being then reduced to a point. The space of functions $\mathcal{O}_{E}$ is the exterior algebra on the dual $\Lambda\left(E^{\prime}\right)$, and the superalgebra of vector fields on $E$ is the space of $E$-valued multilinear maps on $E$, so $\operatorname{Vect}(E)=\Lambda\left(E^{\prime} ; E\right)=\Lambda\left(E^{\prime}\right) \otimes E$. 
The algebraic operations are then straightforwardly defined: if $\eta \in \mathcal{O}_{E}$ and $\alpha \otimes x \in$ $V$ ect $(E)$, operation (1) reads as

$$
(\eta, \alpha \otimes x) \rightarrow \eta \wedge \alpha \otimes x
$$

while operation (2) (graded Lie derivative) gives

$$
(\alpha \otimes x, \eta) \rightarrow L_{\alpha \otimes x}(\eta)=\alpha \wedge i_{x} \eta
$$

where $i_{x}$ denotes the inner product of vectors on exterior forms. One deduces from the formula that if $\alpha$ has degree $|\alpha|$ as exterior form, then vector field $\alpha \otimes x$ has degree $|\alpha|-1$ as graded derivation. It is easy to check that the graded commutator of those graded Lie derivatives satisfies:

$$
\left[L_{\alpha \otimes x}, L_{\beta \otimes y}\right]=L_{\{\alpha \otimes x, \beta \otimes y\}},
$$

the latter bracket being Nijenhuis-Richardson bracket, given by the following formula:

$$
\{\alpha \otimes x, \beta \otimes y\}=i(\alpha \otimes x)(\beta \otimes y)-(-1)^{(|\alpha|+1)(|\beta|+1)} i(\beta \otimes y) \alpha \otimes x
$$

or

$$
\{\alpha \otimes x, \beta \otimes y\}=\alpha \wedge i_{x} \beta \otimes y+(-1)^{|\alpha||\beta|+|\alpha|+|\beta|} \beta \wedge i_{y} \alpha \otimes x .
$$

The graded Lie algebra $\operatorname{Vect}(E)$ is then the Nijenhuis-Richardson algebra [16], wellknown for its applications in deformation theory of Lie algebras; its bracket can be defined axiomatically as the canonical graded extension of the Lie bracket (given by commutator) on $E^{\prime} \otimes E=\mathfrak{g l}(E)$. The relation (3) of compatibility can be proved directly from the above formula, by a tedious but direct computation:

$$
L_{\alpha \otimes x}(\eta \wedge \beta \otimes y)=\left(L_{\alpha \otimes x}(\eta)\right) \beta \otimes y+(-1)^{|\eta| \mid(|\alpha|-1)} \eta \wedge\{\alpha \otimes x, \beta \otimes y\}
$$

So we have obtained an explicit example of a graded Lie-Rinehart algebra.

We can now describe its enveloping algebra; the graded algebra associated to it satisfies $\operatorname{gr} \mathcal{U}\left(\mathcal{O}_{E}, \operatorname{Vect}(E)\right)=S_{\mathcal{O}_{E}}^{* \text { super }}(\operatorname{Vect}(E))$, as mentioned in the previous part. As a $\mathcal{O}_{E}-$ module $V e c t(E)$ is generated by $E$, so it is purely odd and $S^{*, \text { super }}$ reduces to $\Lambda^{*}$ in that case. So one has

$$
\operatorname{gr} \mathcal{U}\left(\mathcal{O}_{E}, \operatorname{Vect}(E)\right)=\mathcal{O}_{E} \otimes \Lambda^{*}(E)=\Lambda^{*}(E) \otimes \Lambda^{*}\left(E^{\prime}\right),
$$

which is graded-commutative. The enveloping algebra $\mathcal{U}\left(\mathcal{O}_{E}, V e c t(E)\right)$ is then a non graded-commutative deformation of $\Lambda^{*}(E) \otimes \Lambda^{*}\left(E^{\prime}\right)$ : this is precisely the relation between differential operators and their symbols. One can interpret this deformation 
in the following way: consider the direct sum of $E$ with its dual, so let $\mathbb{E}=E \oplus E^{\prime}$, and let $<,>$ be the symmetric non degenerate bilinear form on $\mathbb{E}$ defined by duality, so: $\langle x+\alpha, y+\beta\rangle=\alpha(y)+\beta(x)$. We can extend the form $<,>$ to an odd Poisson bracket on $\Lambda^{*}(\mathbb{E})$. Finally, one can check that our algebra of differential operators in dimension $0 \mid n$ turns out to be isomorphic to Clifford algebra $\mathcal{C}(\mathbb{E},<,>)$. See [12] for results of that kind.

Remark: An analogous construction in the purely even case leads to consider the symmetric algebra $S^{*}\left(E^{\prime}\right)$ as the space of polynomial functions on $E$; then $V e c t(E)$ is the space of formal vector fields on $E$, and $\left(S^{*}\left(E^{\prime}\right), \operatorname{Vect}(E)\right)$ is a Lie-Rinehart algebra, whose enveloping algebra is the algebra of formal differential operators on $E$. The latter can also be obtained through a well-known Lie theoretic construction: on $\mathbb{E}=E \oplus E^{\prime}$ let's consider the canonical symplectic (instead of symmetric) form $\varpi(x+\alpha, y+\beta)=\alpha(y)-\beta(x)$, which yields the Heisenberg Lie algebra, and the algebra of differential operators on $E$, or Weyl algebra, is obtained as a quotient of its envelopping algebra.

\section{The case of $\tilde{V}$}

We begin with a more precise definition; let $\tau_{V} \rightarrow V$ be the tangent bundle of $V$, and let $\Lambda_{*} \tau_{V} \rightarrow V$ be the exterior algebra bundle associated to it. Then $\Omega_{*}(V)$ can be identified with its space of sections $\Gamma\left(V, \Lambda_{*} \tau_{V}\right)$, in other words the space of antisymmetric contravariant smooth tensor fields. Then $\Omega_{*}(V)$ is an associative graded commutative algebra for the exterior product $\wedge$ of those tensor fields: if $|A|$ denotes the degree of the tensor field $A \in \Omega_{*}(V)$, one has:

$$
\begin{gathered}
A \wedge(B \wedge C)=(A \wedge B) \wedge C \\
A \wedge B=(-1)^{|A||B|} B \wedge A
\end{gathered}
$$

One has moreover a Lie superalgebra structure, where $\Omega_{*}(V)$ acts onto itself by graded derivations through Schouten bracket ${ }^{2}[$,$] , one has \Omega_{1}(V)=V$ ect $(V)$ and Schouten bracket can then be defined as the unique graded extension of Lie bracket of vector fields, so $\Omega_{*}(V) \subset \operatorname{Der}\left(\Omega_{*}(V)\right)$.

One must take care of degree shifting, a tensor $A \in \Omega_{|A|}(V)$ has degree $|A|$ for associative product, but degree $|A|-1$ as a derivation through Schouten bracket (think of the case of vector fields!); let's now summarize its properties:

1. Graded antisymmetry: $[B, A]=-(-1)^{(|A|-1)(|B|-1)}[A, B]$

\footnotetext{
${ }^{2}$ This bracket should be called Schouten-Nijenhuis, we shall say Schouten for short, but the name of Albert Nijenhuis (1926-2015)[15][10] will appear in the sequel for different brackets
} 


\section{Graded Jacobi identity: $\sum_{(A, B, C)}(-1)^{(|A|-1)(|C|-1)}[A,[B, C]]=0$ \\ 3. Graded Leibniz rule: $[A, B \wedge C]=[A, B] \wedge C+(-1)^{|B|(|A|-1)} B \wedge[A, C]$}

The third formula expresses compatibility between the two operations, more precisely Schouten bracket with $A$ is a derivation of degree $|A|-1$ of $\mathcal{O}_{\tilde{V}}=\Omega_{*}(V)$, as remarked above. To avoid confusion between the two operations we shall use degree shifting; let $k[1]$ be the graded $k$-module with $k$ in degree 1 and 0 elsewhere. Setting $\Omega_{*}(V)[1]=$ $\Omega_{*}(V) \otimes k[1]$ shifts the degree by one.

So the pair $(A, L)$ associated to $\tilde{V}$ will be $\left(\Omega_{*}(V), \Omega_{*}(V)[1]\right)$; one sees at once that one has a super Lie-Rinehart structure, since compatibility axiom (3') turns out to be exactly graded Leibniz rule.

\section{The case of $\hat{V}$}

In the formulas below, the bracket [,] will be reserved for the graded commutator, except perhaps the good old Lie bracket of vector fields on a (non super-)manifold when there will be no ambiguity. We shall use different notations for other brackets: $\{$,$\} for Nijenhuis-Richardson, and \llbracket, \rrbracket$ for Frölicher-Nijenhuis.

In order to identify $\operatorname{Vect}(\hat{V})$ we shall consider the space of vector valued differential forms on $V$, denoted by $\Omega^{*}\left(V, \tau_{V}\right)$. We shall show that those forms act on $\Omega^{*}(V)$ by a natural generalization of the action of vector fields, by inner product or Lie derivative. We shall denote, by a slight abuse of notation, a generic element $X \in \Omega^{*}\left(V, \tau_{V}\right)$ by $X=\alpha \otimes x$ with $\alpha \in \Omega^{*}(V)$ and $x \in V e c t(V)$ (rigorously speaking, this is valid only locally and after some summation, but extension to the general case doesn't add difficulties, since we can use partitions of unit in order to glue local pieces together).

Now $X=\alpha \otimes x$ acts on $\eta \in \Omega^{*}(V)$ by inner product as:

$$
(X, \eta) \rightarrow i(X) \eta=\alpha \wedge i(x) \eta .
$$

One checks immediately that $i(X)$ is a graded derivation of degree $|i(X)|=|\alpha|-1$, so $\Omega^{*}\left(V, \tau_{V}\right)[1]$ is a space of derivations of $\Omega^{*}(V)$. A well-known derivation of $\Omega^{*}(V)$ is exterior derivative $d$, and one defines Lie derivative with respect to $X$ by intertwining inner product and exterior derivative as in the case of vector fields:

$$
\mathcal{L}_{X}=[i(X), d],
$$

where the bracket here denotes simply the graded commutator. If one defines by $I \in \Omega^{*}\left(V, \tau_{V}\right)$ the element satisfying $I(a)=I d\left(T_{a} V\right)$ for any $a \in V$, then one has $i(I) \eta=|\eta| \eta$, and $\mathcal{L}_{I}=d$. 
In the general case, the explicit formula is the following :

$$
\mathcal{L}_{X} \eta=\alpha \wedge L_{x} \eta+(-1)^{|\alpha|} d \alpha \wedge i(x) \eta .
$$

As a graded derivation one has $\left|\mathcal{L}_{X}\right|=|\alpha|$, so with Lie derivative $\Omega^{*}\left(V, \tau_{V}\right)$ is a space of derivations of $\Omega^{*}(V)$. One can furthermore generalize the classical Cartan formulas for Lie derivative and inner product. One has:

1. $[i(X), i(Y)]=i(\{X, Y\})$

2. $\left[\mathcal{L}_{X}, i(Y)\right]=i(\llbracket X, Y \rrbracket)+(-1)^{|X||Y|+1} \mathcal{L}_{i(X) Y}$

3. $\left[\mathcal{L}_{X}, \mathcal{L}_{Y}\right]=\mathcal{L}_{\llbracket X, Y \rrbracket}$

It is now easy to check the first equality, the action of $X$ through $i(X)$ being purely algebraic, fibrewise tensorial; from $X \in \Omega^{k}\left(V, \tau_{V}\right)$, one deduces for each $a \in V$ that $X(a) \in \Lambda^{k} T_{a} V^{*} \otimes T_{a} V$, and so the action of $i(X)$ identifies with the dim $0 \mid n$ case as developed in part (4). Finally the bracket $\{$,$\} identifies with Nijenhuis-Richardson$ bracket on each fiber:

$$
\{X, Y\}(a)=\{X(a), Y(a)\}
$$

Formula (2) and (3) define in fact a new graded Lie bracket, known as FrölicherNijenhuis bracket:

$$
\begin{gathered}
\Omega^{k}\left(V, \tau_{V}\right) \times \Omega^{l}\left(V, \tau_{V}\right) \rightarrow \Omega^{k+l}\left(V, \tau_{V}\right) \\
(X, Y) \rightarrow \llbracket X, Y \rrbracket,
\end{gathered}
$$

it can also be characterized as the unique graded Lie bracket which extends the Lie bracket on $V e c t(V)=\Omega^{0}\left(V, \tau_{V}\right)$ to $\Omega^{*}\left(V, \tau_{V}\right)$.

Moreover, one has a structure of graded $\Omega^{*}(V)$-module on $\Omega^{*}\left(V, \tau_{V}\right)$ defined as $(\eta, \alpha \otimes x) \rightarrow(\eta \wedge \alpha) \otimes x$.

One can also define Frölicher-Nijenhuis bracket by the following explicit formula, if $X=\alpha \otimes x$ and $Y=\beta \otimes y$, then :

$\llbracket \alpha \otimes x, \beta \otimes y \rrbracket=\alpha \wedge \beta \otimes[x, y]+\alpha \wedge \mathcal{L}_{x} \beta \otimes y-\mathcal{L}_{y} \alpha \wedge \beta \otimes x+(-1)^{|\alpha|}(d \alpha \wedge i(x) \beta \otimes y+i(y) \alpha \wedge d \beta \otimes x)$, cf.[10] p.70 sqq.

This bracket plays a key role in integrability of almost complex structures through Newlander-Nirenberg theorem, and also in integrability of multihamiltonian systems in finite dimension[11].

The Lie superalgebra of vector fields on $\hat{V}$ is then characterized by the following: 
Proposition (Frölicher and Nijenhuis, cf.[10][5]):

$$
\operatorname{Vect}(\hat{V})=\operatorname{Der}\left(\mathcal{O}_{\hat{V}}\right)=\Omega^{*}\left(V, \tau_{V}\right)[1] \oplus \Omega^{*}\left(V, \tau_{V}\right),
$$

the derivations being defined as follows: $(A, X) \in \Omega^{*}\left(V, \tau_{V}\right)[1] \oplus \Omega^{*}\left(V, \tau_{V}\right)$ acts on $\eta \in \Omega^{*}(V)$ as $\mathfrak{L}(A, X) \eta=i(A) \eta+\mathcal{L}_{X} \eta$.

One can now compute the commutator, by checking on test differential forms and using formulas of the appendix:

$[\mathfrak{L}(A, X), \mathfrak{L}(B, Y)]=\mathfrak{L}\left(\{A, B\}+\llbracket X, B \rrbracket+(-1)^{p q+1} \llbracket Y, A \rrbracket, i(A) Y+(-1)^{p q+1} i(B) X+\llbracket X, Y \rrbracket\right)$

if $|(A, X)|=p$ and $|(B, Y)|=q$.

That Lie superalgebra will be denoted by $\mathcal{F N}^{*}(V)$, with $\mathcal{F N}^{p}(V)=\Omega^{p+1}\left(V, \tau_{V}\right)[1] \oplus$ $\Omega^{p}\left(V, \tau_{V}\right)$. It admits a natural structure of graded module on the graded commutative associative algebra $\mathcal{O}_{\hat{V}}=\Omega^{*}(V)$, defined by the following formula :

$$
\lambda .(A, X)=\left(\lambda \wedge A+(-1)^{|\lambda|+|X|} d \lambda \wedge X, \lambda \wedge X\right),
$$

for any $\lambda \in \mathcal{O}_{\hat{V}}$.

One then remarks immediately that $\Omega^{*}\left(V, \tau_{V}\right)[1]$ is a sub $\mathcal{O}_{\hat{V}}$-module of $\mathcal{F} \mathcal{N}^{*}(V)$.

Remark : As remarked by the authors in [10] p.72 the commutator formula above looks like a reciprocal semidirect product of Frölicher-Nijenhuis and NijenhuisRichardson algebras, but is more intricated; in [13] the author considers the relevant structure for that kind of algebra, which he calls knit product. Moreover, $\Omega^{*}\left(V, \tau_{V}\right)$ is not a sub $\mathcal{O}_{\hat{V}}$-module of $\mathcal{F} \mathcal{N}^{*}(V)$, as we can easily see from the formula of the action of $O_{\hat{V}}$; one has a short exact sequence of $O_{\hat{V}}$-modules, which doesn't split :

$$
\Omega^{*}\left(V, \tau_{V}\right)[1] \rightarrow \mathcal{F N}^{*}(V) \rightarrow \Omega^{*}\left(V, \tau_{V}\right)
$$

So we have two natural candidates for Lie-Rinehart structures associated to $\hat{V}$ :

1. $(A, L)=\left(\mathcal{O}_{\hat{V}}, \Omega^{*}\left(V, \tau_{V}\right)[1]\right)$

2. $(A, L)=\left(\mathcal{O}_{\hat{V}}, \mathcal{F} \mathcal{N}(V)\right)$

Case 1 is easy, and one has the following

Proposition 6.1: $\left(\mathcal{O}_{\hat{V}}, \Omega^{*}\left(V, \tau_{V}\right)[1]\right)$ with the operations given above defines a Lie-Rinehart structure on the supermanifold $\hat{V}$.

The proof is now straightforward, since it is enough to rewrite the computations of part 4 in a fibrewise version, the superspace being $T_{a} V$ for every $a \in V$. Moreover, 
one can consider the bundle $\tau V \oplus \tau V^{*} \rightarrow V$, equipped with the naturally defined symmetric non degenerate bilinear form, then one has the Clifford bundle associated to that data $\operatorname{Cliff}(V) \rightarrow V$. The algebra of differential operators on $\left(\mathcal{O}_{\hat{V}}, \Omega^{*}\left(V, \tau_{V}\right)[1]\right)$ is then isomorphic to the space of sections of that Clifford bundle.

The case 2. leads to much more complicated calculations, but one has:

Proposition 6.2: The pair $\left(\mathcal{O}_{\hat{V}}, \mathcal{F} \mathcal{N}(V)\right)$ is a Lie-Rinehart algebra.

\section{$7 \quad$ Appendix}

We shall give here some useful formulas for brackets in the Lie superalgebra $\mathcal{F} \mathcal{N}(V)$. If $X \in \Omega^{p}\left(V, \tau_{V}\right)$ and $Y \in \Omega^{q+1}\left(V, \tau_{V}\right)$, one has

$$
\left[\mathcal{L}_{X}, i(Y)\right]=i(\llbracket X, Y \rrbracket)+(-1)^{p q+1} \mathcal{L}_{i(X) Y},
$$

which is equivalent to:

$$
\left[i(Y), \mathcal{L}_{X}\right]=\mathcal{L}_{i(X) Y}+(-1)^{p} i(\llbracket Y, X \rrbracket) .
$$

Those formulas can be found in [10].

Moreover, the bracket $\llbracket, \rrbracket$ doesn't induce a structure of a Lie Rinehart algebra, the obstruction of axiom (3') being satisfied, will be called by analogy with physics literature, the anomaly; it plays a key role in the proof of proposition (6.2) above. The formula of the anomaly is the following:

$$
\llbracket \lambda \wedge X, Y \rrbracket=\lambda \wedge \llbracket X, Y \rrbracket+(-1)^{b(a+l)} \mathcal{L}_{Y} \lambda \wedge X+(-1)^{a+l} d \lambda \wedge i(X) Y
$$

\section{Acknowledgments}

This work gives a partial answer to a question of Sofiane Bouarroudj at EuroMaghreb conference in Algebra, Geometry and Lie theory, in Monastir (Tunisia), in march 2016. The author thanks him and all the organizers as well, and also Friedrich Wagemann for pointing the relevance of [14][9] for the problem.

\section{References}

[1] M. Batchelor. Graded manifolds and vector bundles: a functorial correspondence. J. Math. Phys., 26(7):1578-1582, 1985.

[2] F. A. Berezin and D. A. Leı̆tes. Supermanifolds. Dokl. Akad. Nauk SSSR, 224(3):505-508, 1975. 
[3] Sophie Chemla. Operations for modules on Lie-Rinehart superalgebras. Manuscripta Math., 87(2):199-223, 1995.

[4] Pierre Deligne and John W. Morgan. Notes on supersymmetry (following Joseph Bernstein). In Quantum fields and strings: a course for mathematicians, Vol. 1, 2 (Princeton, NJ, 1996/1997), pages 41-97. Amer. Math. Soc., Providence, RI, 1999.

[5] E. Gozzi and D. Mauro. A new look at the Schouten-Nijenhuis, FrölicherNijenhuis, and Nijenhuis-Richardson brackets. J. Math. Phys., 41(4):1916-1933, 2000 .

[6] Johannes Huebschmann. Poisson cohomology and quantization. J. Reine Angew. Math., 408:57-113, 1990.

[7] Johannes Huebschmann. Lie-Rinehart algebras, Gerstenhaber algebras and Batalin-Vilkovisky algebras. Ann. Inst. Fourier (Grenoble), 48(2):425-440, 1998.

[8] Johannes Huebschmann. Duality for Lie-Rinehart algebras and the modular class. J. Reine Angew. Math., 510:103-159, 1999.

[9] Bas Janssens and Friedrich Wagemann. To be published.

[10] Ivan Kolář, Peter W. Michor, and Jan Slovák. Natural operations in differential geometry. Springer-Verlag, Berlin, 1993.

[11] Y. Kosmann-Schwarzbach and F. Magri. Lax-Nijenhuis operators for integrable systems. J. Math. Phys., 37(12):6173-6197, 1996.

[12] Bertram Kostant and Shlomo Sternberg. Symplectic reduction, BRS cohomology, and infinite-dimensional Clifford algebras. Ann. Physics, 176(1):49-113, 1987.

[13] Peter W. Michor. Knit products of graded Lie algebras and groups. In Proceedings of the Winter School on Geometry and Physics (Srní, 1989), number 22, pages 171-175, 1990.

[14] Karl-Hermann Neeb and Malihe Yousofzadeh. Current superalgebras and unitary representations. arXiv:170\%.00282v1, 2017.

[15] Albert Nijenhuis. Geometric aspects of formal differential operations on tensors fields. In Proc. Internat. Congress Math. 1958, pages 463-469. Cambridge Univ. Press, New York, 1960.

[16] Albert Nijenhuis and R. W. Richardson, Jr. Commutative algebra cohomology and deformations of Lie and associative algebras. J. Algebra, 9:42-53, 1968. 
Bulletin de la Société Royale des Sciences, Vol. 89, Articles, 2020, p. 186 - 197

[17] George S. Rinehart. Differential forms on general commutative algebras. Trans. Amer. Math. Soc., 108:195-222, 1963.

[18] Ping Xu. Gerstenhaber algebras and BV-algebras in Poisson geometry. Comm. Math. Phys., 200(3):545-560, 1999. 